\title{
RECURRENT EXPLOSIVE ERUPTIONS AND THE “SIGMOID-TO-ARCADE” TRANSFORMATION IN THE SUN DRIVEN BY DYNAMICAL MAGNETIC FLUX EMERGENCE
}

\author{
V. ARChONTIS ${ }^{1,2}$, A. W. Hood ${ }^{2}$, AND K. Tsinganos ${ }^{3}$ \\ ${ }^{1}$ Section of Astrophysics, Astronomy and Mechanics, Department of Physics, University of Athens, \\ Panepistimiopolis 15784, Athens, Greece; va11@st-andrews.ac.uk \\ ${ }^{2}$ School of Mathematics and Statistics, St. Andrews University, St. Andrews, KY169SS, UK \\ ${ }^{3}$ National Observatory of Athens, Lofos Nymphon, Thissio, Athens, Greece \\ Received 2014 February 27; accepted 2014 April 4; published 2014 April 22
}

\begin{abstract}
We report on three-dimensional MHD simulations of recurrent mini coronal mass ejection (CME)-like eruptions in a small active region (AR), which is formed by the dynamical emergence of a twisted (not kink unstable) flux tube from the solar interior. The eruptions develop as a result of the repeated formation and expulsion of new flux ropes due to continuous emergence and reconnection of sheared field lines along the polarity inversion line of the AR. The acceleration of the eruptions is triggered by tether-cutting reconnection at the current sheet underneath the erupting field. We find that each explosive eruption is followed by reformation of a sigmoidal structure and a subsequent "sigmoid-to-flare arcade" transformation in the AR. These results might have implications for recurrent CMEs and eruptive sigmoids/flares observations and theoretical studies.
\end{abstract}

Key words: Sun: activity - Sun: coronal mass ejections (CMEs) - Sun: magnetic fields

Online-only material: color figures

\section{INTRODUCTION}

In the active Sun, the emergence of magnetic flux from the solar interior can lead to the formation of active regions (ARs; e.g., Zwaan 1985), which are often associated with coronal mass ejections (CMEs), flaring activity, and other dynamic events (see, e.g., van Driel-Gesztelyi \& Culhane 1995; Schrijver 2009; Archontis 2012). In addition, observational studies (see, e.g., Hudson et al. 1998; Canfield et al. 1999) have revealed that ARs with an S-shaped morphology are the most favorable candidates for eruptive activity in the Sun. The term "sigmoid" has been used by Rust \& Kumar (1996) to denote the overall (forward or reverse) S-shaped structure of an AR. Observations (see, e.g., Sterling et al. 2000; Pevtsov 2002; Liu et al. 2010) have also shown that eruptive ARs, which initially display a degree of twist adopting a sigmoidal shape, evolve into a post-eruption flare arcade that consists of field lines with a cusp-like shape. This process is known as "sigmoid-to-arcade" evolution and it could happen repeatedly (e.g., Gibson et al. 2002; Nitta \& Hudson 2001) in some ARs: a bright (EUV/X-ray) sigmoid is reformed after each CME(-like) eruption, which is accompanied by a hot arcade underneath it.

Numerical experiments have demonstrated the importance of magnetic flux emergence for driving CME-like eruptions associated with flaring activity in emerging flux regions (EFRs; e.g., Shibata \& Magara 2011; Kusano et al. 2012). Simulations have also shown the formation of sigmoidal structures in EFRs in conjunction with the (partial) eruption of magnetic flux ropes (e.g., Gibson et al. 2006; Archontis et al. 2009). The onset of recurrent eruptions has been studied in the context of a break-out magnetic scenario (e.g., DeVore \& Antiochos 2008) and in EFRs (e.g., reconnection between the emerging and a pre-existing magnetic field, e.g., Archontis \& Hood 2008; MacTaggart \& Hood 2009). The repeated "sigmoid-toarcade" evolution associated with CMEs has been shown by the kinematically driven (quasi-static) emergence of a highly twisted magnetic torus into a pre-existing potential coronal field (Chatterjee \& Fan 2013). In this Letter, we present a three-dimensional MHD experiment of the onset of recurrent CME-like eruptions driven by the dynamical emergence of a horizontal twisted flux tube in a highly stratified atmosphere. We find that the "sigmoid-to-arcade" process occurs naturally due to reconnection of sheared field lines during the eruptions.

\section{THE MODEL}

Cartesian geometry, using the Lare $3 \mathrm{~d}$ code (Arber et al. 2001). Viscous and Ohmic heating are considered through shock viscosity and Joule dissipation. Uniform explicit resistivity is included, with $\eta=10^{-3}$. Initially, the plasma is embedded into a plane-parallel hydrostatic atmosphere. A sub-photospheric, adiabatically stratified layer resides in the range $(-7.2 \mathrm{Mm} \leqslant z<$ $0 \mathrm{Mm}$ ). The layer above, at $0 \mathrm{Mm} \leqslant z<2.3 \mathrm{Mm}$, which is isothermal $(5100 \mathrm{~K})$ at the beginning before the temperature increases with height up to $\approx 4 \times 10^{4} \mathrm{~K}$, is mimicking the photosphere/chromosphere. The layer at $2.3 \mathrm{Mm} \leqslant z \leqslant 3.1 \mathrm{Mm}$ represents the transition region. An isothermal coronal layer $(\approx 1 \mathrm{MK})$ is included at $3.1 \mathrm{Mm}<z \leqslant 57.6 \mathrm{Mm}$. The initial magnetic field is a horizontal twisted flux tube at $z_{0}=-2.1 \mathrm{Mm}$ oriented along the positive $Y$-axis. The axial field of the cylindrical tube is defined by

$$
B_{y}=B_{0} \exp \left(-r^{2} / R^{2}\right), \quad B_{\phi}=\alpha r B_{y},
$$

where $R=450 \mathrm{~km}$ is the radius of the tube, $r$ is the radial distance from the tube axis $\left(r^{2}=x^{2}+\left(z+z_{0}\right)^{2}\right)$, and $\alpha=$ $0.0023 \mathrm{~km}^{-1}$ is the uniform twist around the axis of the tube, which is stable to the kink instability. A density deficit is applied along the axis of the tube, making its central part more buoyant than its footpoints:

$$
\Delta \rho=\left[p_{t}(r) / p(z)\right] \rho(z) \exp \left(-y^{2} / \lambda^{2}\right),
$$

where $p_{t}$ is the pressure within the flux tube and $\lambda$ defines the length of the buoyant part of the tube. We use $\lambda=0.9 \mathrm{Mm}$ and 


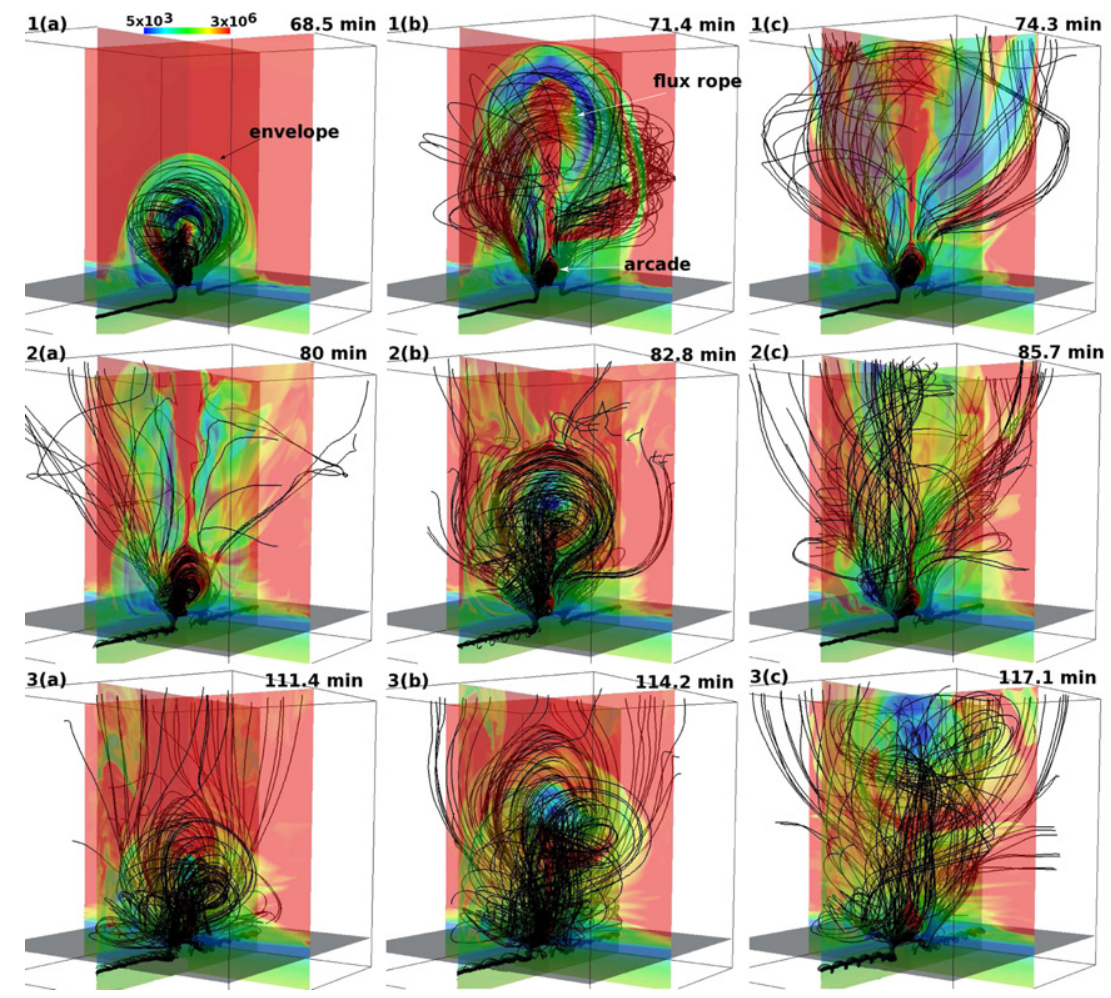

Figure 1. Temperature and magnetic field topology during the eruptions. Shown here are the two vertical midplanes and the horizontal slice at the base of the photosphere.

(A color version of this figure is available in the online journal.)

an initial field strength for the tube that corresponds to plasma $\beta \approx 14$. The numerical domain is $[-32.4,32.4] \times[-32.4$, $32.4] \times[-7.2,57.6] \mathrm{Mm}$ in the longitudinal $(x)$, transverse $(y)$, and vertical $(z)$ directions, respectively. The grid has 420 nodes in all directions with periodic boundary conditions in $y$. Open boundary conditions have been implemented along $x$ and at the top of the numerical domain, allowing for the outflow of plasma. The bottom boundary is a non-penetrating, perfectly conducting wall.

\section{RESULTS AND DISCUSSION}

Our experiment shows the recurrent eruptive behavior of an EFR during $4.5 \mathrm{hr}$ of its evolution. Figure 1 shows the temperature distribution together with magnetic field lines, which have been traced from the footpoints of the sub-photospheric flux tube. Figure 1(a) shows the cool adiabatic expansion of the emerging field. The outermost field lines have already emerged into the corona to form an envelope (ambient) field for the magnetic flux, which continues to emerge from the solar interior. Part of this magnetic flux forms a new magnetic flux rope that erupts into the outer solar atmosphere in an ejective manner opening the envelope field (Figures 1(b) and (c)). There are at least four ejective eruptions during the evolution (three of them are shown in Figure 1) of the EFR, displaying several common characteristics, such as: (a) the marked expansion of the erupting magnetized volume, (b) the transport of dense (lowatmospheric) material to the high atmosphere, (c) the explosive heating of the plasma underneath the erupting core of the field, and (d) the formation of a hot arcade with a cusp-like shape in the low atmosphere.

The formation of a new flux rope and the driving mechanism of its eruption in similar flux emergence experiments have been studied in previous simulations (e.g., Magara \& Longcope 2001; Manchester et al. 2004; Archontis \& Török 2008; Archontis \& Hood 2012). Figure 2 (panels (a)-(c)) is a synoptic illustration of the formation and rise of the first new flux rope in the present experiment. At $t=62.8$ minutes (Figure 2(a)), the emerging field lines (blue and cyan), which have previously undergone shearing, reconnect with each other to form two new sets of field lines: the white field lines that (will) constitute the central part of the developing flux rope and the yellow field lines that stay behind and do not erupt. The red field lines represent the envelope field. At $t=68.5$ minutes ((Figure 2(b)), the white field lines are traced from the center of the cross section of the erupting flux rope, at the vertical $(x z)$ midplane. The blue and cyan field lines continue to reconnect, forming new field lines: the orange field lines, which wrap around the footpoints of the white field lines, developing a magnetic flux rope structure, and the yellow field lines, which form an arcade. Note that now the envelope field lines are stretched out so that they are about to reconnect in a tether-cutting manner underneath the flux rope. At $t=71.4$ minutes ((Figure 2(c)), the well-developed flux rope consists of straight (white) field lines along its axis and twisted field lines (blue/cyan/orange/red) around its axis. An important result is that at this stage of the evolution, the envelope field lines reconnect in a tether-cutting manner at a strong and thin current layer under the rope. This process triggers the ultimate ejective eruption of the flux rope toward the outer solar atmosphere. Therefore, in this case, the release of the downward tension of the envelope field lines, which under certain conditions can halt the ejective eruption (e.g., Archontis \& Hood 2012), is not due to external reconnection with a pre-existing coronal magnetic field (e.g., Archontis \& Török 2008) in a break-out manner, but by tether-cutting internal reconnection underneath the erupting plasma. 

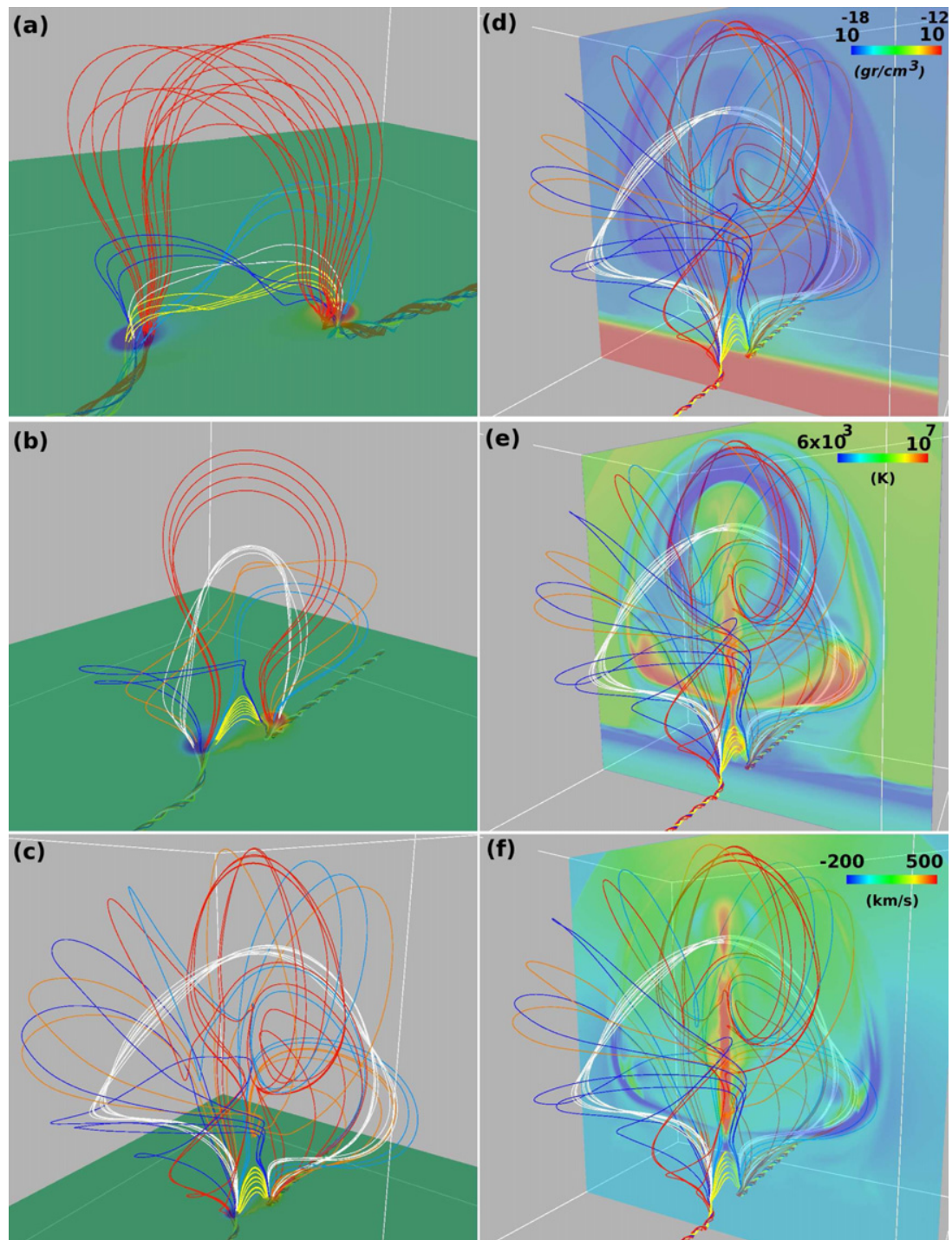

Figure 2. Field line topology during the first eruption (panels (a)-(c)). The horizontal slice shows the Bz distribution at the photosphere (red;positive, blue;negative) within the range $[-500,500] \mathrm{G}$. Times are $t=62.8$ minutes, $t=68.5$ minutes, and $t=71.4$ minutes for panels (a)-(c), respectively. Logarithmic density and temperature, and vertical velocity distribution at the vertical $(x z)$ midplane are shown in panels (d)-(f) at $t=71.4$ minutes.

(A color version of this figure is available in the online journal.)

Figure 2(d) shows that the eruption of the flux rope brings dense plasma from the low atmosphere into the corona. The heavy material $(\approx 1-2$ orders heavier than the background plasma) is accumulated at the dips of the twisted field lines of the flux rope. Figure 2(e) shows that the ejective phase of the eruption is characterized by the cool adiabatic expansion of the field and the heating of the plasma due to tether-cutting reconnection of the field lines. Figure 2(f) shows that highspeed $\left(\approx 800 \mathrm{~km} \mathrm{~s}^{-1}\right)$ bi-directional jets are emitted vertically (upward/downward) from the reconnection site underneath the rising flux rope. The upward reconnection jet adds momentum to the rising flux rope and heats the plasma inside the erupting volume. Therefore (Figure 2(e)), cool (purple) and hot (yellow/red) plasma is found to be expelled together with the erupting core of the flux rope. In the first two eruptions (which are faster; see also Figure 3), the top edge of the upward jet nearly reaches to the core of the erupting field. Due to its high speed, it deforms the concave part of the twisted field lines that surrounds the erupting core from below. The downward reconnection jet collides with the top (flux pile-up) regime of the arcade forming a termination shock. As a result, the plasma is compressed locally at high temperatures, causing flaring of the arcade.

Eventually, a second flux rope is formed at the upper photosphere/chromosphere, as new field lines emerge and undergo shearing and reconnection (such as the blue and cyan field lines in Figures 2(a)-(c)). The innermost field lines of the arcade undergo the same process, and thus they are incorporated into the formation of the flux rope. The outermost field lines of the arcade constitute a new envelope field for the second flux rope to rise into. Initially, the flux rope rises slowly (with a speed of about $40 \mathrm{~km} \mathrm{~s}^{-1}$ until $t=80$ minutes) and then it erupts in a fast-rise phase, accelerating to a velocity of $\approx 280 \mathrm{~km} \mathrm{~s}^{-1}$ in the next $90 \mathrm{~s}$. The onset of acceleration is accompanied by tether-cutting reconnection of the new envelope's field lines.

The following eruptions occur in a slightly different manner. The magnetic field at the center of the EFR does not become strong enough to emerge above the photosphere with the standard $\Omega$-loop-like configuration. On the contrary, it spreads out horizontally and the magnetic pressure increases in the vicinity of the two main polarities of the EFR. There, the 

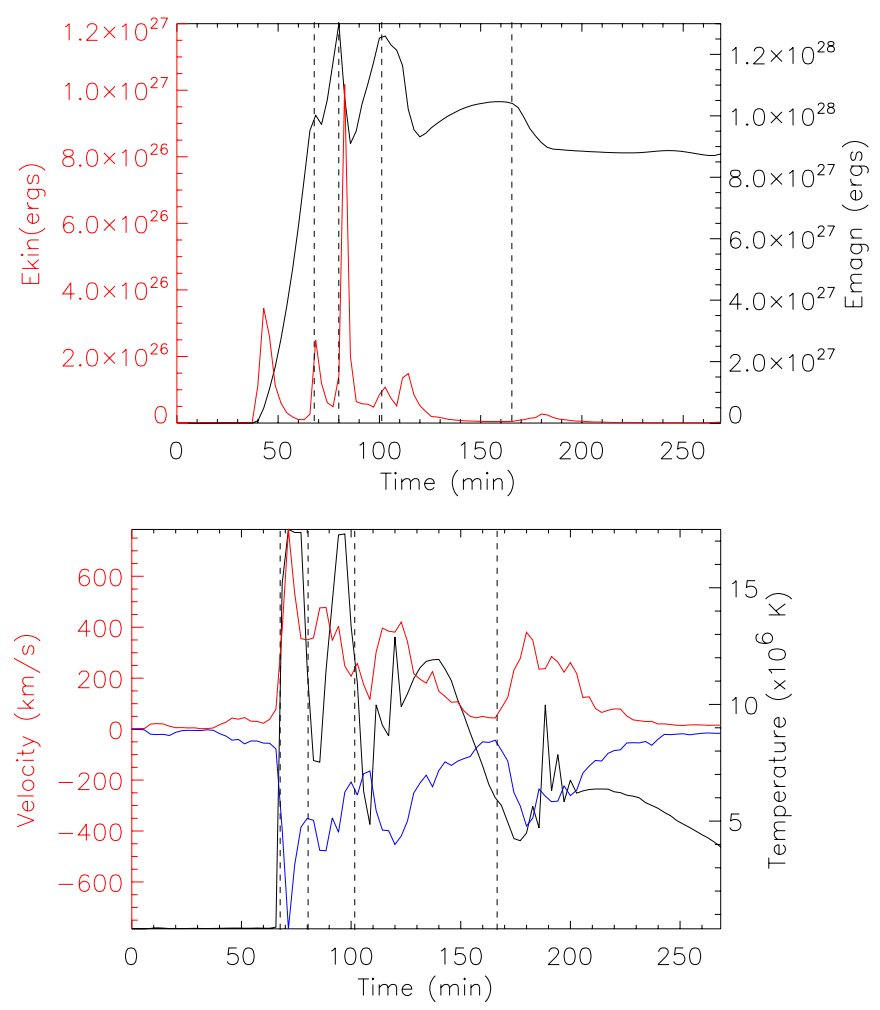

Figure 3. Top: temporal evolution of the magnetic (black line) and kinetic (red) energy above the photosphere. Bottom: temporal evolution of the maximum positive/negative (red/blue, respectively) $V_{\mathrm{z}}$ and maximum temperature (black) above the photosphere.

(A color version of this figure is available in the online journal.)

magnetic field lines can emerge to form two magnetic lobes, similar to the emergence of a weakly twisted emerging field (Archontis et al. 2013). The lateral expansion of the magnetic lobes brings their field lines into contact at the center of the EFR and causes them to reconnect, forming another flux rope. The eruption of the flux rope is driven by the Lorentz force, which is eventually enhanced by tether-cutting reconnection of the envelope field lines.

In summary, the tether-cutting reconnection following the first eruption restructures the magnetic configuration in the solar atmosphere, so that the post-eruption state resembles the preeruption state of the first eruptive event (Figure 2(a)). Eventually, the emergence of new magnetic flux and the associated shearing inject free magnetic energy into the system initiating a second eruption very similar (homologous) to the first one. Overall, the sequence of the homologous eruptions is attributed to (1) ongoing flux emergence, shearing, and reconnection in the low atmosphere (e.g., up to the transition region) and (2) to the tether-cutting reconnection in the corona and the dynamical reconfiguration of the system to a state similar to the initial one.

There are two differences in the topology of the field and the dynamics prior to the eruptions. First, the envelope field in the initial eruption consists of the uppermost emerging field lines, while in the following eruptions it is formed by the field lines of the flare arcade. Second, the formation of the erupting flux rope in the first two eruptions occurs due to shearing and reconnection of the emerging field at the center of the AR. In the other two eruptions, the emerging field develops two side magnetic lobes (see the work by Archontis et al. 2013), which eventually reconnect, forming the magnetic flux rope. Despite these differences, the key processes responsible for the driving of the homologous eruptions in our experiment are similar in all events.

Figure 3 (top) shows the temporal evolution of the total magnetic and kinetic energy above the photosphere. The four eruptions start to occur at the times marked by the vertical dashed lines. In each eruption, magnetic energy drops and kinetic energy increases rapidly. The first increase of the kinetic energy ( $t \approx 40$ minutes) corresponds to the initial emergence of the magnetic field. The second eruption starts while the lower part (i.e., where tether-cutting reconnection of the envelope field lines occurs) of the first eruption is still within the numerical domain. For example, at $t \approx 83$ minutes, the tether-cutting reconnection upflow (jet) of the first eruption originates at $z \approx 44 \mathrm{Mm}$, and thus the corresponding total kinetic energy resembles the accumulated energy of the first two eruptions. After the first eruption, the decrease (increase) of the magnetic (kinetic) energy becomes lower with each of the following eruptions, which indicates that the CME-like eruptions become progressively less energetic. This is because there is a certain amount of flux and energy available to the system for driving the eruptions. This energy comes from the sub-photospheric magnetic flux tube. Due to dynamical emergence, the flux is eventually exhausted, and thus the eruptions produced are less energetic.

Note that the total magnetic energy increases after each eruption. This increase is important for the build-up of the necessary amount of energy that is required for the onset of the successive eruptions. In the first and second eruptions, the increase is due to the dynamical emergence and the shearing of the magnetic field around the center of the EFR. In the following eruptions, shearing is less pronounced but emergence continues to occur, which leads to the formation of the two magnetic lobes. Eventually, the system reaches a quasi-static equilibrium and the magnetic energy saturates.

Figure 3 (bottom) shows the temporal evolution of the vertical component of the velocity field (maximum and minimum value) and the temperature (maximum) above the photosphere. Strong bi-directional flows are emitted during each eruption. These flows are the reconnection jets, which originate in the current sheet underneath the erupting flux rope. There is a very good correlation between the upflows and the downflows during the evolution of the system: for every upflow there is a corresponding downflow with almost the same magnitude. The fastest upflow occurs in the first eruption $\left(\approx 800 \mathrm{~km} \mathrm{~s}^{-1}\right)$. In the other eruptions, the associated jets run with progressively lower speeds, within the range $450-350 \mathrm{~km} \mathrm{~s}^{-1}$. The measured velocity at the center of the erupting flux ropes, in the last snapshot before they exit the domain and while they are accelerated, is between $\approx 200-250 \mathrm{~km} \mathrm{~s}^{-1}$. This indicates that the reconnection jets can indeed add momentum to the erupting plasma volume.

The evolution of the temperature shows that each eruptive event is followed by intense plasma heating. In a similar manner to the temporal evolution of $V_{\mathrm{z}}$, the maximum heating decreases over time. It drops from $17 \mathrm{MK}$ after the first eruption to about $10 \mathrm{MK}$ in the last eruptive event. Therefore, the heating decreases when the eruption is less energetic. Intense heating occurs at the apex of the cusp-like arcade underneath the reconnection site of the produced jets. Therefore, each eruption is followed by a flaring episode. The time offset between the onset of the eruption and the heating of the arcade is determined by the time that the downward reconnection jet takes to reach the arcade and heat it. 


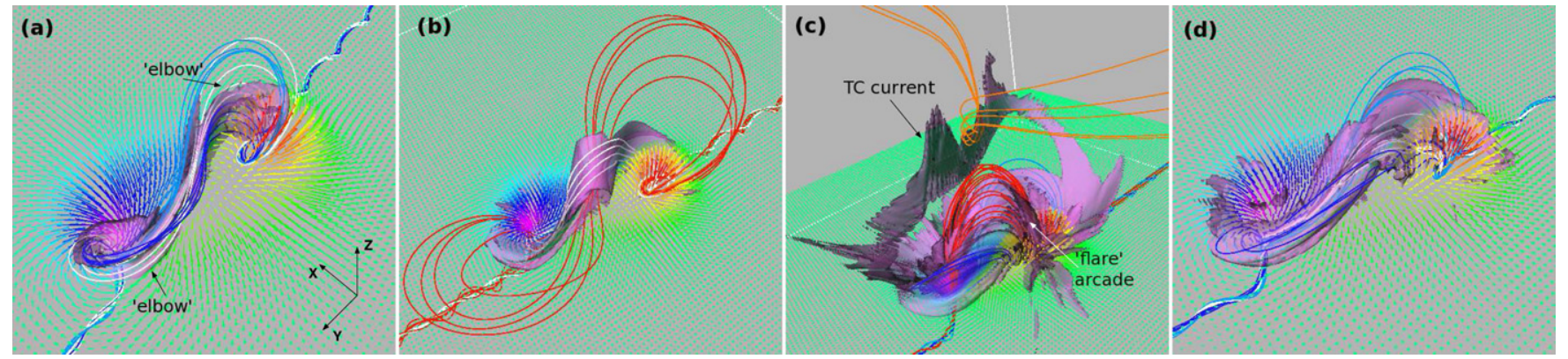

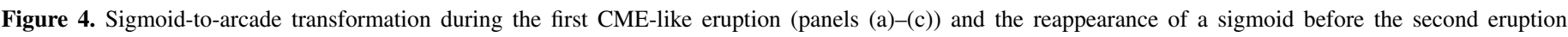

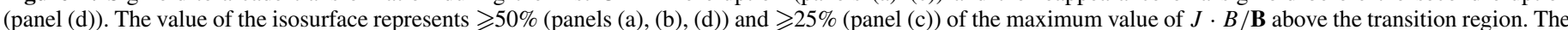

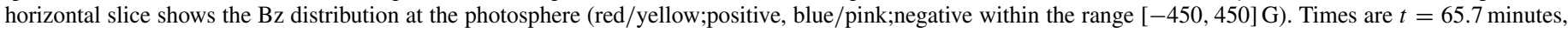
$t=68.5$ minutes, $t=71.4$ minutes, and $t=77.2$ minutes for panels (a)-(d), respectively.

(A color version of this figure is available in the online journal.)

The recurrent CME-like eruptions are associated with the appearance of sigmoidal structures in the EFR. Figure 4 shows the three-dimensional visualization of the isosurface $J \cdot B / \mathbf{B}$, where $J$ is the electric current and $B$ is the magnetic field strength. At $t=65.7$ minutes (panel (a)), the isosurface and the field lines that are traced from this structure adopt an overall S-like shape associated with the twist and writhe of the rising magnetic field. Some of these field lines (white) are dipped at the center of the sigmoid and expand toward its two ends ("elbows"). There are also sheared field lines (blue/cyan) that surround only one of the "elbows," and thus they make only a half turn along the sigmoid. As these field lines come closer together, the current becomes large at their interface (along the polarity inversion line), developing the sigmoidal structure. Eventually, the blue and cyan field lines will reconnect around the center of the EFR to form a new flux rope. Due to reconnection, we expect that the sigmoid's field lines will be heated to high temperatures. At this stage of the simulation, we find that the dense plasma of the sigmoidal structure has an average temperature of $O\left(5 \times 10^{5}\right) \mathrm{K}$ over the integrated volume (i.e., above the transition region) of the isosurface, and hence it may appear as a bright S-shaped structure at the solar atmosphere.

At $t=68.5$ (panel (b)), the sigmoid develops a loop-like structure at its center. This is the current-currying flux rope that starts to rise, leading to the partial eruption of the sigmoid. The (white) field lines, which have been traced from the loop, belong to the erupting flux rope. The red field lines have been traced from the center of the sigmoidal isosurface underneath the flux rope. The flaring of the plasma, which follows the eruption, starts from this central area of the sigmoid. As the eruption proceeds (panel (c)), a new thin and curved current layer is formed underneath the erupting plasma. This is where tether-cutting reconnection occurs (TC current). Thus, field lines (orange), which were previously part of the envelope field, now reconnect, adopting a V shape at the center of the current layer. Under the TC current, the downward-reconnected field lines (red) form the "flare" arcade, which is heated to more than 15 MK. Thus, the bright arcade does not appear to be part of the pre-flare sigmoid but it is a new structure associated with the tethercutting reconnection. The (blue/cyan) field lines are traced from the lower-lying remnants of the sigmoid, which temporarily fades away. However, shortly after the first eruption (panel (d)), the dynamical emergence and the shearing of the field continue to operate, leading to the reappearance of a sigmoidal structure (blue/cyan/white field lines have been traced in a similar manner to panel (a)). A similar process (i.e., sigmoid-to-arcade conversion) is repeated during the following eruptions.

We have performed a preliminary parametric study, which shows that the parameters of the system may affect its evolution and dynamics. For instance, for larger values of plasma $\beta$ or $\lambda$ of the sub-photospheric flux tube, the eruptions occur at later times. In addition, we find that for an initial field strength of the tube that corresponds to $\beta \geqslant 39$, the eruptions are confined. The ejection of helicity and the reformation of sigmoids for a wider range of parameters will be presented in a forthcoming work.

The simulations were performed on the STFC- and SRIFfunded UKMHD cluster at the University of St. Andrews. The authors acknowledge support by EU (IEF-272549 grant) and the Royal Society. V.A. and A.W.H. are grateful for indepth discussions during the ISSI workshop "Magnetic flux emergence in the solar atmosphere" in Bern.

\section{REFERENCES}

Arber, T., Longbottom, A., Gerrard, C., \& Milne, A. 2001, JCoPh, 171, 151 Archontis, V. 2012, RSPTA, 370, 3088

Archontis, V., \& Hood, A. W. 2008, ApJL, 674, L113

Archontis, V., \& Hood, A. W. 2012, A\&A, 537, A62

Archontis, V., Hood, A. W., Savcheva, A., Golub, L., \& Deluca, E. 2009, ApJ, 691,1

Archontis, V., Hood, A. W., \& Tsinganos, K. 2013, ApJ, 778, 42

Archontis, V., \& Török, T. 2008, A\&A, 492, L35

Canfield, R. C., Hudson, H. S., \& McKenzie, D. E. 1999, GeoRL, 26, 627

Chatterjee, P., \& Fan, Y. 2013, ApJL, 778, L8

DeVore, C. R., \& Antiochos, S. 2008, ApJ, 680, 740

Gibson, S. E., Fan, Y., Török, T., \& Kliem, B. 2006, SSRv, 124, 131

Gibson, S. E., Fletcher, L., Del Zanna, G., et al. 2002, ApJ, 574, 1021

Hudson, H. S., Lemen, J. R., St. Cyr, O. C., Sterling, A. C., \& Webb, D. F. 1998, GeoRL, 25, 2481

Kusano, K., Bamba, Y., Yamamoto, T. T., et al. 2012, ApJ, 760, 31

Liu, R., Liu, C., Wang, S., Deng, N., \& Wang, H. 2010, ApJL, 725, L84

MacTaggart, D., \& Hood, A. W. 2009, A\&A, 508, 445

Magara, T., \& Longcope, D. W. 2001, ApJL, 559, L55

Manchester, W., IV, Gombosi, T., DeZeeuw, D., \& Fan, Y. 2004, ApJ, 610, 588

Nitta, N. V., \& Hudson, H. S. 2001, GeoRL, 28, 3801

Pevtsov, A. A. 2002, SoPh, 207, 111

Rust, D. M., \& Kumar, A. 1996, ApJL, 464, L199

Schrijver, C. J. 2009, AdSpR, 43, 739

Shibata, K., \& Magara, T. 2011, LRSP, 8, 6

Sterling, A. C., Hudson, H. S., Thompson, B. J., \& Zarro, D. M. 2000, ApJ, 532,628

van Driel-Gesztelyi, L., \& Culhane, J. L. 1995, SSRv, 144, 351

Zwaan, C. 1985, SoPh, 100, 397 